\title{
Diseño de macro y metacontingencia para la promoción del comportamiento proambiental y urbano responsable
}

\author{
Macro and meta-contingency design for the promotion of a \\ responsible pro-environmental and urban behavior
}

\author{
Pablo Páramo $1 \underline{\mathrm{ORCID}}$, Andrea Burbano $2 \underline{\mathrm{ORCID}}$ \\ 1 Universidad Pedagógica Nacional \\ ${ }^{2}$ Universidad Colegio Mayor de Cundinamarca \\ Colombia
}

Fecha correspondencia:

Recibido: abril 13 de 2020.

Aceptado: marzo 9 de 2021.

Forma de citar:

Páramo, P., \& Burbano, A.

(2021). Diseño de macro y

metacontingencia para la promoción

del comportamiento proambiental y

urbano responsable. Rev. CES Psico,

14(1), 36-48.

\section{Open access}

(c) Copyright

Licencia creative commons

Etica de publicaciones

Revisión por pares

Gestión por Open Journal System

DOl: http://dx.doi.org/10.21615/

cesp.14.1.4

ISSN: 2011-3080

Comparte

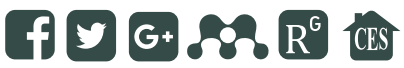

\section{Resumen}

El objetivo de este trabajo teórico es introducir los conceptos de macrocontingencia y metacontingencia en el campo de la psicología ambiental y su papel en la promoción de acciones colectivas en pro del medio ambiente natural y construido. Se proponen este tipo de contingencias para el diseño de prácticas culturales y programas sociales que ayuden a resolver problemas ambientales como el cambio climático, la extinción de especies, la contaminación del agua, la deforestación, las migraciones, el vandalismo, etc., e incentivar comportamientos urbanos responsables como el uso de la bicicleta y del transporte urbano, el reciclaje, etc. Aunque las acciones de cada persona pueden contribuir a las soluciones, el planteamiento del presente manuscrito se centra en el efecto acumulativo y las acciones entrelazadas de los individuos que producen un resultado eficaz para el bienestar de muchas personas y del medio ambiente. La conducta guiada por reglas se presenta como el mecanismo de aprendizaje para introducir las macrocontingencias y metacontingencias en la comunidad. A partir del pragmatismo como visión paradigmática, se discute la efectividad de las macrocontingencias y metacontingencias para explicar los procesos culturales en la promoción de acciones efectivas para el medio ambiente.

Palabras clave: Macrocontingencia, Metacontingencia, Psicología Ambiental, Cambio Cultural, Comportamientos Urbanos Responsables, Comportamiento Proambiental, Comportamiento Sostenible.

\section{Abstract}

This article aims to introduce the concepts of macrocontingency and metacontingency into environmental psychology to promote collective actions in pro of the natural and urban environment. The design of cultural practices oriented to achieve the goal requires the definition of contingencies that promote social process to solve environmental problems such as: climate change, species extinction, water contamination, deforestation, migration, vandalism, etc, and to encourage responsible urban behaviors such as the 


\section{Sobre los autores:}

1. Ph.D. en Psicología (Environmental). Magíster en Ciencia. Coordinador del Grupo de Investigación Pedagogía Urbana y Ambiental. Profesor Titular Doctorado Interinstitucional en Educación, Universidad Pedagógica Nacional.

2. Ph.D. en Estudios Territoriales. Magíster en Gestión Urbana. Arquitecta. Profesora Facultad de Ingenieria y Arquitectura, Universidad Colegio Mayor de Cundinamarca. use of bicycles, urban transport, recycling, among others. Although each person's actions may contribute to the solutions, the approach of this theoretical paper is focused on the cumulative effect and the interconnected actions of people that produce an effective outcome for the well-being of many and the environment. Rule - driven behavior is showed as the learning mechanism to introduce the macrocontingencies and metacontingencies in the community. Based on pragmatism as a paradigmatic view, it is discussed the effectiveness of macrocontingencies and metacontingencies in explaining cultural processes to promote effective actions for the environment.

Keywords: Macrocontingency, Metacontingency, Environmental Psychology, Cultural Change, Responsible Urban Behaviors, Sustainable Behavior, Pro-Ecological Behavior.

\section{Introducción}

Gran parte de la investigación en psicología ambiental se ha orientado a buscar soluciones a los problemas ambientales, identificando, por una parte, algunos atributos de los individuos como su personalidad, orientación política, credo religioso, nivel educativo, género, actitudes, etc., a partir de los cuales se puedan predecir conductas como el reciclaje, el uso de fuentes de energía no renovable, eliminación del consumo de plástico y del consumo en general (Barazarte, Neaman, Vallejo, \& García, 2014; Guifford, 2014), a las que se les ha denominado comportamiento proambiental o conductas protectoras del ambiente (Corral-Verdugo, 2002). Por otra parte, interveniendo sobre el comportamiento o a través de la exposición directa de las personas a los ambientes naturales, apoyada en la generación de procesos mediadores entre el individuo y el ambiente como la biofilia (Wilson, 1993), la conectividad con la naturaleza (Corraliza \& Collado, 2011; Olivos-Jara, Aragonés, \& Navarro-Carrascal, 2013), la Acción Razonada (Ajzen \& Fishbein, 1973), la Acción Planeada (Ajzen, 1991; 2005), la teoría de la Activación de la Norma (Schwartz, 1977) y la conducta normativa de Foco Normativo (Cialdini et al., 1990). Para confirmar la existencia de los atributos que actúan como mediadores del cambio observado, las investigaciones se han valido principalmente de cuestionarios. Se exceptúan de estas tendencias, los estudios que buscan modificar el comportamiento individual, a partir de la programación de reforzadores o castigos a las conductas observadas, deseables o indeseables, respectivamente; trabajos que fueron populares en las décadas del 70 y 80 (Bachman \& Kattzev, 1982; Chapman \& Risley, 1974; Geller, 1982, 1987; Geller, Winett, \& Everett, 1982 ). No obstante, los avances y aportes de estas investigaciones, tanto unos como otros acercamientos han sido cuestionados en su efectividad para alcanzar el cambio esperado (Geller, 2002; Gifford \& Sussman, 2012; Schultz \& Kaiser 2012; Uzzell, 1999), por lo que es indispensable explorar otras aproximaciones teóricas que puedan explicar los resultados que se han obtenido y orientar de manera más eficaz el comportamiento deseado.

En consecuencia, introducimos en este artículo los conceptos de macrocontingencia y metacontingencia, por el papel pragmático que puedan jugar en la creación de programas sociales orientados a promover prácticas culturales deseables para la protección del medio ambiente natural y construido.

Desde la perspectiva psicológica, el término contingencia es entendido como una relación de dependencia entre eventos, o como un resultado probable, a partir de una condición y acción previa que establece una determinada consecuencia positiva o negativa en una conducta particular de un individuo (Ribes, 1990), y por tratarse de la conducta de varios individuos que hacen parte de un grupo social se le anteponen los prefijos "macro" o "meta" para referirse a aquellas relaciones de dependencia 
Pág 38

Para resolver eficazmente los problemas del ambiente natural y construido, es indispensable partir de la condición social de los seres humanos, quienes al vivir en comunidad y en un ambiente en crisis requieren aprender formas de actuación que les permitan regular la interacción con los demás y con los elementos del ambiente de forma responsable; es decir, aprender comportamientos individuales y en cooperación que puedan ser sostenibles en el tiempo y en consecuencia se conviertan en prácticas culturales. entre una conducta y una situación, mediante las cuales se busca obtener un resultado, ya sea que se demande o no la acción interdependiente entre varios individuos para recibir una consecuencia positiva o negativa (Glenn, 1998).

\section{Comportamientos proambientales y urbanos responsables}

Además de los comportamientos proambientales, en los que tradicionalmente se ha enfocado la psicología ambiental, tales como el reciclaje, la reducción del consumo de plástico o de carne, o pagar más por un producto ecológico, en el entorno urbano se requiere otro tipo de acciones que incluyen la relación con otros individuos, principalmente extraños, en un ambiente construido en el que figuran los equipamientos urbanos y los espacios públicos. En el ambiente urbano se busca primordialmente que los individuos hagan uso de transporte público y de la bicicleta como alternativas al vehículo particular, se abrochen el cinturón de seguridad, usen adecuadamente los espacios públicos, cuiden los monumentos patrimoniales, no fumen en espacios donde hay otras personas, crucen las calles por las zonas demarcadas para los peatones, actúen en solidaridad con quien esté en dificultad, eviten entrar en contacto con otras personas cuando padezcan de una enfermedad contagiosa, participen en la toma de decisiones que afectan a la ciudadanía y al medio ambiente en general, y sigan las reglas de convivencia, entre otras acciones, las cuales se han venido agrupando bajo el concepto Comportamientos Urbanos Responsables (CUR) (Páramo, 2010; 2013).

Para resolver eficazmente los problemas del ambiente natural y construido, es indispensable partir de la condición social de los seres humanos, quienes al vivir en comunidad y en un ambiente en crisis requieren aprender formas de actuación que les permitan regular la interacción con los demás y con los elementos del ambiente de forma responsable; es decir, aprender comportamientos individuales y en cooperación que puedan ser sostenibles en el tiempo y en consecuencia se conviertan en prácticas culturales.

\section{Prácticas culturales}

Se entiende como prácticas culturales aquellas formas de actuación similar, aprendidas y mantenidas por los integrantes de un grupo en un contexto similar, las cuales pueden ser transmitidas socialmente de una generación a otra mediante un proceso de selección por consecuencias, como el que explica la evolución de las especies y del comportamiento, que conduce a la evolución cultural (Harris, 1979). Las consecuencia de las prácticas culturales se refieren a aquellos eventos ambientales contingentes a las conductas entrelazadas y a la sumatoria de los resultados que esto conlleva, lo que afecta la probabilidad de ocurrencia de la unidad cultural que finalmente es seleccionada. La mayoría de los comportamientos hacen parte de una práctica cultural adquirida por cualquier individuo que se vincula a un grupo social, ya sea en su condición de niño o extraño, mediante procesos de aprendizaje que involucran el modelamiento, el seguimiento de reglas verbales o mediante la exposición a contingencias del ambiente (Todorov, 2013).

Con el fin de lograr que los comportamientos proambientales se conviertan en prácticas culturales sostenibles es necesario resolver varios interrogantes: ¿qué se requiere para que los comportamientos de los individuos contribuyan a mejorar el medio ambiente y la convivencia, y sean adoptados por el grueso de la ciudadanía y sostenibles en el tiempo? ¿Qué se necesita para que las personas actúen de forma cooperativa en función de proteger el ambiente natural y urbano, y para que esta 
Al planificar acciones que pretenden un cambio cultural en las que participan grupos de individuos, el foco de interés debe ponerse en: el número de personas que contribuyen a alcanzar los resultados; la variedad de formas de actuación que ayudan a producir el cambio esperado; la meta de la intervención; y, la programación de consecuencias. forma de actuación se extienda en el tiempo y pasen de una generación a otra? En otras palabras, ¿qué se debe hacer para que la protección del medio ambiente se constituya en una práctica cultural? La respuesta a estos interrogantes se puede conseguir a partir del diseño de macrocontingencias y metacontingencias (Glenn, 2003, 2004; Ostrom, 2009).

\section{Dos conceptos teóricos para la comprensión de los compor- tamientos proambientales y urbanos responsables}

\section{Las macrocontingencias ambientales}

Al planificar acciones que pretenden un cambio cultural en las que participan grupos de individuos, el foco de interés debe ponerse en: el número de personas que contribuyen a alcanzar los resultados; la variedad de formas de actuación que ayudan a producir el cambio esperado; la meta de la intervención; y, la programación de consecuencias.

Una opción para alcanzar este cambio es mediante el diseño de una macrocontingencia, entendida como una relación entre la conducta periódica de varios individuos y el producto resultante de la sumatoria de las acciones de los individuos quienes hacen su aporte de manera individual (Houmanfar \& Rodríguez, 2006). Ejemplo de una macrocontingencia que propicia CUR, es la aplicación de multas a conductores de vehículos que no respetan el paso de peatones en una intersección vial de alta accidentalidad, cuya sumatoria o agregado produce un mayor número de conductores que ceden el paso a los peatones y una reducción en los niveles de accidentalidad, y en la que los individuos que intervienen son inespecíficos. En este sentido, Van Houten y Malefant (2004) evaluaron el comportamiento de un grupo de conductores respecto a los peatones después de implementar un programa que incluyó estrategias como peatones señuelo, volantes de retroalimentación, advertencias escritas y verbales, e infracciones policiales. Los resultados mostraron que el número de conductores que cedieron el paso a los peatones se incrementó tras la introducción del programa; resultados que se mantuvieron al hacerles seguimiento durante un año gracias a que permanecieron las condiciones de la estrategia.

Por su parte, un estudio realizado en California reportó una asociación positiva entre un programa de control del tabaco a gran escala con la reducción del tabaquismo y, además, logró la reducción de los gastos de atención médica personal (Lightwood, Dinno, \& Glantz, 2008).

Zandecki (2012) examinó el comportamiento de reciclar mediante la implementación de avisos informativos y de retroalimentación ubicados encima de 26 contenedores de desechos en una Universidad de la Florida. Los letreros informativos comunicaban sobre qué es y dónde poner el material de reciclaje, su efecto en el ambiente y otros mensajes como: "Las botellas de vidrio tardan más de 4.000 años en descomponerse"; y los de "retroalimentación" informaban sobre la cantidad de material reciclable y no reciclable que se depositaba en los contenedores cada día. Ambos tipos de letreros se ubicaron solos y combinados en distintos lugares. Los datos sugieren que ambas intervenciones por separado aumentaron las conductas de reciclaje, y al combinarlas se presentó aún mayor nivel de reciclaje; y la intervención con letreros de retroalimentación por sí sola mostró mayor aumento en el reciclaje que con letreros informativos. 
Pág 40

Propuestas de programas como el de la alcaldía de Bogotá (Colombia) que proponen una prima para los taxistas asociada a la reducción de accidentes, o el proyecto de ley que cursan en el congreso de la República de Colombia que busca reducir el valor del seguro obligatorio de vehículos según los niveles de accidentalidad o infracciones del conductor el año anterior, son también ejemplos de diseño de macrocontingencias mediante las que se espera beneficiar a un grupo, por cuanto pueden disminuir los niveles de accidentalidad general y mejorar la movilidad.
Propuestas de programas como el de la alcaldía de Bogotá (Colombia) que proponen una prima para los taxistas asociada a la reducción de accidentes, o el proyecto de ley que cursan en el congreso de la República de Colombia que busca reducir el valor del seguro obligatorio de vehículos según los niveles de accidentalidad o infracciones del conductor el año anterior, son también ejemplos de diseño de macrocontingencias mediante las que se espera beneficiar a un grupo, por cuanto pueden disminuir los niveles de accidentalidad general y mejorar la movilidad.

Un programa de gobierno implementado en Brasilia (Brasil), descrito por Lé Sénéchal-Machado y Todorov (2017), se constituye en un ejemplo de diseño de macrocontingencias. Este programa reunió el esfuerzo del gobierno, universidades, medios de comunicación y ONG's para promover el uso del paso peatonal. El gobierno desarrolló campañas publicitarias para mejorar el comportamiento de conductores y peatones, los medios de comunicación duplicaron sus publicaciones sobre el tráfico de la ciudad de Brasilia y las ONG's, realizaron marchas en las calles promoviendo los comportamientos apropiados en la movilidad tanto de peatones como de conductores. Por su parte, la Universidad de Brasilia organizó un grupo permanente de discusión sobre los problemas de tráfico y la Policía persistió en la aplicación de la ley que da prioridad a los peatones y enseñó a los peatones a detenerse, mirar a ambos lados, esperar que los vehículos frenen y cruzar solo por el paso peatonal. Este programa logró que prácticamente todos los conductores se detuvieran ante las personas en el paso peatonal y este comportamiento se constituyó en una práctica cultural, que se mantiene en parte porque el gobierno pinta y señaliza los nuevos cruces peatonales y continúa organizando campañas, los medios trabajan para enseñar las reglas y persiste la educación de conductores y peatones.

En esta misma línea es importante destacar el impacto que tuvo el programa de cultura ciudadana que se implementó en la ciudad de Bogotá (Colombia) durante la alcaldía de Anthanas Mockus a finales de los años 90. Bajo la premisa "todos ponen", se invitaba a los ciudadanos a asumir compromisos con la ciudad y a defender el patrimonio común, instigando a los peatones a cruzar por las zonas demarcadas y a los conductores de los vehículos a detenerse en estas zonas, promoviendo el pago de impuestos y aportes voluntarios, e implementando la estrategia denominada la "hora zanahoria", que prohibía la venta de licor entre la 1: 00 a.m. y las 6:00 a.m., entre otras medidas. El programa se valió de mimos, tarjetas de reconocimiento social y otros elementos simbólicos, cuyos resultados produjeron gran entusiasmo y lograron cambiar el comportamiento de los ciudadanos, principalmente, al comienzo y durante su implementación, sin embargo, fueron decayendo con el paso del tiempo como se vino registrando en los medios de comunicación (Revista Semana, 2016) y en los programas de medición de opinión como "Bogotá Cómo Vamos" (Oróstegui, s.f.), lo cual se puede atribuir a la falta de continuidad en la aplicación de las contingencias debido a los cambios de administración.

El problema con este tipo de programas de acuerdo con los planteamientos que se vienen exponiendo, es, que a menos que las autoridades penalicen con frecuencia el incumplimiento o destaquen permanentemente los logros alcanzados, el procedimiento probablemente pierda algo de efectividad, por lo que, para fortalecer estas relaciones y su estabilización en el tiempo, es necesario normatizar la regla, promoverla dentro de la ciudadanía y hacerle seguimiento. 
Una metacontingencia se establece cuando hay una relación funcional entre una práctica cultural que tiene tradición en un grupo de personas que actúan de manera interdependiente para mantener el comportamiento entre sus miembros y conseguir un resultado, el cual se convierte en objeto de selección, aumentando la probabilidad de que la conducta cooperativa se repita y se transmita a la siguiente generación. Por lo que se espera que los individuos cooperarán entre sí cuando su comportamiento interdependiente produzca mayores consecuencias favorables que mediante su comportamiento independiente.
Es importante advertir que en una macrocontingencia no existe una relación funcional entre las conductas de los individuos en el sentido de que la conducta de una persona se relacione con la de otra, sea reforzándola o censurándola; cada individuo que presenta la conducta está actuando bajo las condiciones de una misma contingencia, sin relación con los otros miembros del grupo, aunque estén contribuyendo a crear condiciones que afectan al grupo en su totalidad (Glenn, 2004).

Si la conducta repetida de varios individuos da como resultado un producto agregado como puede ser la reducción de accidentes o la cantidad de toneladas recicladas, este puede tener un impacto significativo sobre el grupo, sin embargo, no se da lugar a una unidad de selección cultural que trascienda en el tiempo o entre generaciones (Harris,1979), en la medida en que no se está tratando con una totalidad que pueda ser objeto de selección sino con conductas que ocurren de manera independiente. Este tipo de conductas individuales: usar el cinturón de seguridad, evitar accidentes, cruzar por la zona demarcada, disminuir el consumo de carne, reciclar, etc., no están del todo integradas, sino que corresponden a un conjunto de conductas no relacionadas entre individuos, por lo que no ocurrirá necesariamente una selección que se constituya en práctica cultural, dado que afecta únicamente a individuos aislados, contrario a lo que sucede cuando se hace referencia a una metacontingencia.

\section{Las metacontingencias ambientales}

Glenn, Malott, Andery, Benvenuti, Houmanfar, Sandaker, Todorov, Tourinho, y Vasconcelos (2016) definen una metacontingencia como una relación contingente o funcional entre contingencias de comportamiento individuales entrelazadas y recurrentes que dan lugar a un producto agregado, el cual es seleccionado por algún evento o condición ambiental (contar con un ambiente sano, reducción de impuestos, mayor seguridad). Dicho de otra manera, una metacontingencia se establece cuando hay una relación funcional entre una práctica cultural que tiene tradición en un grupo de personas que actúan de manera interdependiente para mantener el comportamiento entre sus miembros y conseguir un resultado, el cual se convierte en objeto de selección, aumentando la probabilidad de que la conducta cooperativa se repita y se transmita a la siguiente generación. Por lo que se espera que los individuos cooperarán entre sí cuando su comportamiento interdependiente produzca mayores consecuencias favorables que mediante su comportamiento independiente.

En la metacontingencia el producto o resultado requiere no solo de la conducta de varios individuos sino de contingencias entrelazadas, repetidas, que mantengan la interrelación entre conductas de personas diferentes (Marques, Leite, \& Benvenuti, 2012; Smith, Houmanfar, \& Louis, 2011). Ejemplos de diseño de metacontingencias: los campesinos de una región se unen para acabar con unos insectos que les están dañando sus cultivos; un grupo de vecinos se reúne para recuperar el parque del barrio, unos lo barren, otros siembran o podan árboles y otros más prestan vigilancia para poder disfrutarlo, y cada participante obtiene sus propios beneficios; de igual forma ocurre una metacontingencia cuando se planean y realizan viajes compartidos para ir al trabajo; o, cuando un equipo de fútbol o un grupo de investigación universitario articula las acciones de sus integrantes, para ganar el partido o posicionar al grupo de investigación al tiempo que van obteniendo beneficios. De esta manera, el producto agregado o la recompensa obtenida tiene doble función: reforzar el comportamiento del participante, a la vez que selecciona las contingencias interdependientes que llevan al resultado final, el cual es finalmente seleccionado. 
Pág 42

De este modo, cuando las conductas interdependientes de los individuos actúan de manera cohesionada para conseguir una consecuencia positiva para el grupo, se da lugar a una metacontingencia, que, a su vez, produce como resultado la selección de un o unos comportamientos o práctica cultural garantizando su sostenimiento en el tiempo. Según este planteamiento, el diseño de una metacontingencia que articule, por ejemplo, las acciones individuales de la industria, el transporte y la acción individual podrá mitigar los efectos del cambio climático al reducir la cantidad de CO2 en la atmósfera.
Para que una macrocontingencia se convierta en una metacontingencia sería necesario, entonces, crear las condiciones entre las conductas individuales y sus efectos, interrelacionándolas, lo cual se consigue normalmente mediante reglas verbales. En las ciudades de Bogotá y Cúcuta en Colombia, Páramo y Páramo (2016) y Páramo y Contreras (2018) estudiaron el efecto de una metacontingencia diseñada a partir de la difusión en el espacio público de reglas que anunciaban metacontingencias para los ciudadanos que adoptaran ciertos CUR, como cruzar por la cebra, caminar por el andén y no por la calzada, detener el vehículo antes de la cebra mientras el semáforo está en rojo, entre otros. Tras levantar una línea de base de los comportamientos observados en las avenidas de estas ciudades, se introdujeron avisos en las calles que explicitaban las reglas a seguir e invitaban al ciudadano a reclamar su cumplimiento y aceptar el llamado de atención de otras personas en caso de no acogerse a la regla. En la ciudad de Cúcuta los investigadores se valieron de una pantalla digital gigante en un punto de alta circulación de los ciudadanos y de pancartas para exhibir semanalmente la cantidad de individuos que acogían las reglas sobre los comportamientos establecidos; mientras que en Bogotá se creó una señalética para difundir las reglas, invitando a los ciudadanos a llamar la atención de quien no las cumpliera y a aceptar el llamado de atención de otro conciudadano, además de informar mediante calcomanías pegadas a la señalética el número de ciudadanos que habían seguido las reglas la semana anterior. Las intervenciones no solo mostraron un aumento en el número de individuos que acataron las reglas, además, se observaron cambios relacionados con otras reglas de la movilidad, evidenciando una generalización de los CUR.

De este modo, cuando las conductas interdependientes de los individuos actúan de manera cohesionada para conseguir una consecuencia positiva para el grupo, se da lugar a una metacontingencia, que, a su vez, produce como resultado la selección de un o unos comportamientos o práctica cultural garantizando su sostenimiento en el tiempo. Según este planteamiento, el diseño de una metacontingencia que articule, por ejemplo, las acciones individuales de la industria, el transporte y la acción individual podrá mitigar los efectos del cambio climático al reducir la cantidad de C02 en la atmósfera.

Pero no siempre las metacontingencias ocurren o se planifican de modo deseable socialmente. El vandalismo de los bienes públicos de la ciudad como los grafitis sobre los monumentos patrimoniales, el daño intencional sobre el mobiliario urbano, las agresiones a la policía, etc., que se presentan durante las protestas, se sabe, no son acciones individuales; son sostenidas por el reconocimiento o coacción de otros individuos que mantiene este tipo de prácticas.

La adopción de una práctica social va a depender, entonces, del diseño de las metacontingencias que defina el grupo social. Esto da lugar a una acción cooperativa, que inicia con la conducta de un individuo $y$, dependiendo del resultado que esta produzca, puede ser imitada y generalizada mediante reglas verbales y generar una práctica cultural, comportamiento compartido y sostenido por los miembros de ese grupo social.

El diseño de metacontingencias garantiza, al igual que la aplicación del concepto de escenarios de comportamiento de Barker (1978), que las prácticas culturales deseables se mantengan a lo largo del tiempo, independientemente de quienes estén presentes; los individuos pueden reemplazarse como ocurre en el paso de una generación a otra, mientras las contingencias prevalezcan, las prácticas sociales se mantendrán. 
El diseño de programas para la creación o transformación de prácticas culturales sobre comportamientos proambientales y urbanos responsables no solo debe especificar el arreglo de las contingencias que regularán el comportamiento deseable o indeseable, sino también determinar cómo deben ser enseñadas y difundidas en la población.
Al diseñar una metacontingencia no se trata únicamente de conseguir un efecto acumulativo de diferentes conductas, también se busca que estas conductas se interrelacionen de modo que contribuyen a un resultado que trascienda a otras generaciones.

Las diversas políticas públicas suscritas en los últimos años frente a la conducta de fumar, por ejemplo, han contribuido a moldear en el público no-fumador una respuesta crítica sobre quien fuma, de manera que ahora hay mayor probabilidad de que un fumador encuentre un no-fumador que lo evite o que le pida que deje de fumar. La combinación de acciones como el incremento del precio de los cigarrillos, la difusión de las consecuencias nocivas para la salud derivadas de su consumo, más las sanciones sociales negativas y legales que reciben los fumadores son, en buena parte, responsables por el descenso marcado en la prevalencia e incidencia de fumadores. En la misma dirección, para contrarrestar la epidemia del coronavirus desatada recientemente, los gobiernos podrían promover en los ciudadanos el aislamiento, mostrando, por ejemplo, el número de infectados o muertos semanalmente en los distintos sectores de la ciudad como resultado del grado en que se acogen a la medida de aislamiento.

\section{Papel de las reglas verbales en el diseño de las macro y metacontingencias}

El diseño de programas para la creación o transformación de prácticas culturales sobre comportamientos proambientales y urbanos responsables no solo debe especificar el arreglo de las contingencias que regularán el comportamiento deseable o indeseable, sino también determinar cómo deben ser enseñadas y difundidas en la población. En este sentido, las reglas verbales, se constituyen en el medio a través del que se transmiten y aprenden las contingencias entre las personas, al especificar las condiciones bajo las cuales se van a producir las consecuencias; de este modo, estas reglas cumplen un papel muy importante en el diseño de las macro y metacontingencias.

Las reglas verbales son descripciones que establecen las relaciones de contingencia entre las ocurrencias de un comportamiento, el comportamiento mismo y las consecuencias que este produce en el ambiente (Catania et al., 1989; Hayes, 1989). En la misma dirección, Glenn (1991) afirma que las reglas describen el funcionamiento y organización de las contingencias, indican qué hacer, cuándo hacerlo y qué sucedería al hacerlo. En el caso de la educación ambiental sobre comportamientos proambientales o urbanos responsables resulta más eficaz enseñar a partir de reglas en la medida en que estas establecen lo que debe hacerse para mejorar las condiciones del ambiente con las consecuencias esperadas, sin necesidad de recurrir a atributos mediacionales como la adopción de normas prescriptivas y descriptivas, actitudes, personalidad, conciencia ambiental, etc. (Páramo, 2017).

Tanto las macro como las metacontingencias pueden ser definidas a partir de las reglas que especifican el tipo de consecuencias que un producto agregado traerá al grupo de personas (Malott \& Glenn, 2006; Todorov, 2010). La regla establecerá que el resultado de las acciones individuales sumadas dará lugar a un beneficio, con lo cual se espera que la acción mancomunada entre los individuos se mantenga a largo plazo. En consecuencia, las campañas educativas y políticas sobre la protección del medio ambiente serán más efectivas cuando se pueda establecer la conexión entre la conducta individual y su efecto acumulativo que equivale a un resultado positivo para el individuo en el caso de las macrocontingencias, y para un grupo de personas en el caso de las metacontingencias. 
Pág 44

Los conceptos de macrocontingencia y metacontingencia son una contribución teórica importante para la psicología ambiental que sirven para orientar el diseño de acciones hacia la cooperación en pro del ambiente natural y construido, y para estudiar e interpretar los programas y experiencias sociales y de gestión urbana que han generado cambios en las prácticas culturales orientadas a la protección del medio ambiente.
Finalmente, muchas de las conductas que conforman una práctica cultural deseable son exhibidas por individuos y se constituyen en una guía hacia la consecuencia favorable e igualmente en una regla a seguir sin necesidad de explicitarla. Por esto, las acciones de varias personas funcionan como modelos de comportamiento para los demás, a la vez que, ofrecen consecuencias positivas, lo que genera una interconexión entre los individuos y da lugar a una práctica cultural sostenida que se puede transmitir a la siguiente generación.

\section{Conclusiones}

Los conceptos de macrocontingencia y metacontingencia son una contribución teórica importante para la psicología ambiental que sirven para orientar el diseño de acciones hacia la cooperación en pro del ambiente natural y construido, y para estudiar e interpretar los programas y experiencias sociales y de gestión urbana que han generado cambios en las prácticas culturales orientadas a la protección del medio ambiente. Ambos conceptos se constituyen en herramientas útiles que permiten comprender la efectividad o no, de ciertos programas y diseñar estrategias para conseguir el cambio del comportamiento deseado y la respectiva transmisión cultural. Sin embargo, como sostiene Zilio (2019), es probable que frente al concepto de metacontingencia no se tenga todavía la madurez suficiente para entender cómo es que evoluciona la cultura, en la medida en que las contingencias no actúan necesariamente sobre un resultado acumulado sino sobre individuos.

Derivado de los planteamientos presentados en este artículo se puede concluir que la acción educativa y el diseño de políticas orientadas al cuidado del ambiente, como ahorro de agua, reciclaje de basuras, uso de la bicicleta o el transporte público, reducción del consumo de carne, dependerá del número de personas involucradas en dichas acciones, de la frecuencia con que se ejecute cada uno de estos comportamientos, del producto agregado que se derive de los mismos y de las consecuencias resultado de estas acciones. Y en el caso de las metacontingencias, dependerá además de la acción cooperativa que asuman los ciudadanos.

En resumen, en el diseño de una macro o metacontingencia ambiental se requiere precisar: 1. ¿Quiénes serían los participantes? 2 ¿Cuál es el comportamiento objetivo y cuál su producto agregado?; 3. ¿Cuáles serían las consecuencias de las acciones planteadas, individuales y colectivas?; 4. ¿Cuáles son las ocasiones más relevantes para que ocurran las diferentes conductas? Y, en el diseño de una metacontingencia 5. ¿Qué estrategia debe implementarse para que los individuos actúen de manera entrelazada para conseguir tanto los beneficios individuales como colectivos?

En el marco de las macro y metacontingencias la identificación de la frecuencia, incidencia o prevalencia de ciertas formas de actuación que conforman las prácticas culturales deseables de cohesión social, permitirán retroalimentar a las personas sobre sus acciones, de tal manera que se favorezca la promoción de dichas prácticas y la generación de cambios perdurables en la manera en que las personas se relacionan entre sí para conseguir el cambio esperado. En este mismo sentido, el diseño de políticas públicas para proteger el medio ambiente deberá tomar en consideración igualmente los beneficios sociales que se obtengan de la acción colectiva, lo que es propio de la visión pragmática de la psicología ambiental (Altman \& Rogoff, 1987), teniendo claro que una macrocontingencia será más eficiente en ambientes amplios, como en los espacios públicos urbanos y una metacontingencia en grupos más pequeños, en donde sus integrantes tengan mayores posibilidades de conocerse entre sí. 
Tanto para el diseño de macro como de metacontingencias es fundamental resaltar los resultados de los comportamientos, y en esta misma vía, las campañas publicitarias y los programas educativos ambientales no solo deben orientarse a incentivar los comportamientos deseados sino a destacar los logros alcanzados por la acción colectiva (Sandoval, 2012), de lo contrario los individuos desconocerán los resultados de sus acciones individuales o mancomunadas, y, en consecuencia, los logros culturales no se mantendrán. Con el diseño de las metacontingencias los individuos entenderán los beneficios de actuar en función del grupo social.
No basta con incentivar conductas deseables mediante campañas publicitarias; es indispensable resaltar los logros alcanzados por la acción individual o cooperativa. Así, por ejemplo, si únicamente se informa a la ciudadanía sobre la reducción de robos de celulares en el sistema de transporte masivo mediante avisos ubicados en los paraderos de buses, esta no sabrá cuál ha sido su papel o el del gobierno, y por lo tanto no tendrá claridad sobre la acción que se le demanda. Será más efectiva la campaña si se menciona el número de denuncias que facilitaron la judicialización de los ladrones, por ejemplo.

Serán más sostenibles los cambios en las formas de actuación de las personas si se anuncian como resultado de las mismas, distintos tipos de consecuencias que puedan beneficiar a diversos individuos. Por ejemplo, si se anuncia que el número de toneladas recicladas en un periodo de tiempo ha contribuido a favorecer a un importante sector de la población que se dedica a esta labor; a proteger el medioambiente; a mejorar la estética de la ciudad; y a reducir enfermedades contagiosas, se tendrá mayor impacto sobre los indicadores del reciclaje, siempre que los individuos respondan a distintos tipos de consecuencias. En la medida que se establezcan y se hagan explícitas tanto las acciones individuales como colectivas y las consecuencias favorables, se podrá conseguir un cambio cultural duradero y sostenible, por cuanto serán los individuos y no los agentes externos o la publicidad quienes se encarguen de regularse entre sí para mantener la práctica cultural. Tanto para el diseño de macro como de metacontingencias es fundamental resaltar los resultados de los comportamientos, y en esta misma vía, las campañas publicitarias y los programas educativos ambientales no solo deben orientarse a incentivar los comportamientos deseados sino a destacar los logros alcanzados por la acción colectiva (Sandoval, 2012), de lo contrario los individuos desconocerán los resultados de sus acciones individuales o mancomunadas, y, en consecuencia, los logros culturales no se mantendrán. Con el diseño de las metacontingencias los individuos entenderán los beneficios de actuar en función del grupo social.

La aproximación a los problemas ambientales mediante las nociones de macrocontingencias y metacontingencia constituye un aporte a la teoría de la psicología ambiental, a la psicología social y a la investigación en estos campos, por cuanto permite comprender e identificar aquellas acciones que han mostrado su efectividad en la promoción y surgimiento de comportamientos proambientales y CUR.

\section{Referencias}

Ajzen, I. (2005). Attitudes, Personality and Behaviour; Open University Press (Second Edition). Mc Graw Hill Education.

Ajzen, I. (1991). The theory of planned behavior. Organizational Behavior and Human Decision Processes, 50(2), 179-211.

Ajzen, I., \& Fishbein, M. (1973). Attitudinal and normative variables as predictors of specific behavior. Journal of Personality and Social Psychology, 27(1), 41-57. https://doi.apa.org/doiLanding?doi=10.1037\%2Fh0034440

Altman, I., \& Rogoff, B. (1987). World views in psychology: Trait, interactional, organismic, and transactional perspectives. In D. Stokols \& I, Altman (Eds.), Handbook of Environmental Psychology. Vol. 1 (pp. 7-40). Wiley.

Bachman, W., \& Kattzev, R. (1982). The effects on non-contingent free bus tickets and personal commitment on urban bus ridership. Transportation Research, 16A, 103-108.

Barazarte, R., Neaman, A., Vallejo, F., \& García, P. (2014). El conocimiento ambiental 
y el comportamiento proambiental de los estudiantes de la Enseñanza media, en la Región de Valparaíso (Chile). Revista de Educación, 364, 12-34. http://dx.doi. org/10.4438/1988-592X-RE-2014-364-255

Barker, R. G. (1978). Habitats, environments, and human behavior: studies in ecological psychology and eco-behavioral science from the Midwest Psychological Field Station, 1947-1972. Jossey-Bass.

Catania, A. C., Shimoff, E., \& Matthews, B. A. (1989). An experimental analysis of rule-governed behavior. In S. C. Hayes (Ed.), Rule-governed behavior: Cognition, contingencies, and instructional control (pp. 119-150). Plenum Press. https://doi. org/10.1007/978-1-4757-0447-1 4

Chapman, C., \& Risley, T.R. (1974). Anti-litter procedures in an urban high-density area. Journal of Applied Behavior Analysis, 7(3), 477-384. https://doi.org/10.1901/ jaba.1974.7-377

Cialdini, R. B., Reno, R. R., \& Kallgren, C. A. (1990). A focus theory of nonnative conduct: Recycling the concept of norms to reduce littering in public places. Journal of Personality and Social Psychology, 58(6), 1015-1026. http://dx.doi. org/10.1037/0022-3514.58.6.1015

Corral-Verdugo, V. (2002). Psicología de la Conservación: El estudio de las conductas protectoras del ambiente. En V. Corral Verdugo (Ed.). Conductas protectoras del Ambiente. CONACYT-UniSon.

Corraliza, J., \& Collado, S. (2011). La naturaleza cercana como moderadora del estrés infantil. Psicothema 23(2), 221-226.

Geller, E. S. (2002). The Challenge of social change: A Behavioral Scientist's Perspective, Social Marketing Quarterly, 8(2), 15-24. https://doi.org/10.1080/15245000212551

Geller, E. S. (1987). Applied Behavior Analysis and Environmental Psychology: From Strange Bedfellows to a Productive Marriage. In D. Stokols \& I. Altman (Eds) Handbook of Environmental Psychology. John Willey \& Sons.

Geller, E.J., Winett, R.A., \& Everett, P.B. (1982). Preserving the environment: New strategies for behavior change, Journal of Leisure Research, 14(4), 365-366. https://doi. org/10.1080/00222216.1982.11969535

Gifford, R. (2014). Environmental Psychology Matters. Annual Review of Psychology, 65(1), 541-579. http://doi.org/10.1146/annurev-psych-010213-115048

Gifford, R., \& Sussman, R. (2012). Environmental attitudes. In S. D. Clayton (Ed.), Oxford library of psychology. The Oxford handbook of environmental and conservation psychology (pp. 65-80). Oxford University Press. https://doi.org/10.1093/ oxfordhb/9780199733026.013.0004

Glenn, S. (2004). Individual Behavior, Culture, and Social Change. The Behavior Analyst, 27(2), 133-151. https://doi.org/10.1007/BF03393175

Glenn, S. (2003) Operant contingencies and the origin of culture. In K.A. Lattal \& P.N. Chase (Eds.), Behavior theory and philosophy (pp 223-242). New York: Klewer Academic/Plenum. https://doi.org/10.1007/978-1-4757-4590-0 12

Glenn, S. (1998). Contingencies and Metacontingencies: Toward a Synthesis of Behavior Analysis and Cultural Materialism. The Behavior Analyst, 11(2), 161-179. doi: $10.1007 / B F 03392470$

Glenn, S. (1991). Contingencies and meta-contingencies: Relations among behavioral, cultural, and biological evolution. In P. A. Lamal (ed.), Behavioral Analysis of Societies and Cultural Practices (pp. 39-73). Hemisphere Press.

Glenn, S., Malott, M., Andery, M. A. P. A., Benvenuti, M., Houmanfar, R., Sandaker, I., ... Vasconcelos, L. (2016). Toward consistent terminology in a behaviorist approach to cultural analysis. Behavior and Social Issues, 25, 11-27. https://doi.org/10.5210/ bsi.v25i0.6634.

Harris, M. (1979). Cultural Materialism: The Struggle for a Science of Culture. Random 
House Eds.

Hayes, S. C. (1989). Rule-governed behavior: Cognition, contingencies, and instructional control. Plenum Press.

Houmanfar, R., \& Rodrigues, N. (2006). The metacontingency and the behavioral contingency: Points of contact and points of departure. Behavior and Social Issues, 15, 13-30. https://doi.org/10.5210/bsi.v15i1.342

Lightwood J.M., Dinno A., \& Glantz S.A. (2008). Effect of the California Tobacco Control Program on personal health care expenditures. PLoS Med 5(8), e178. https://doi. org/10.1371/journal.pmed.0050178

Machado, V.L.S., \& Todorov, J.C. (2017). Crossing the Crosswalk: How to Make a City Walk the Line. Behavior and Social Issues 26, 200-203. https://doi.org/10.5210/ bsi.v26i0.7933

Malott, M. E., \& Glenn, S. S. (2006). Targets of intervention in cultural and behavioral change. Behavior and Social Issues, 15, 31-56. https://doi.org/10.5210/bsi. v15i1.344

Marques, N., Leite, F., \& Lobato Benvenuti, M. (2012). Conceptual and Experimental Directions for Analyzing Superstition in the Behavioral Analysis of Culture. Es/ Rlp, 44(1), 55-63. http://dx.doi.org/10.14349/rlp.v44i1.929

Ostrom, E. (2009). A behavioral approach to the rational choice theory of collective action. The American Political Science Review, 92, 1-22.

Olivos-Jara, P., Aragonés, J. I., \& Navarro-Carrascal, O. (2013). Educación ambiental: itinerario en la naturaleza y su relación con conectividad, preocupaciones ambientales y conducta. Revista Latinoamericana de Psicología, 45(3), 503-513. http:// dx.doi.org/10.14349/rlp.v45i3.1490

Orostegui, O. (s.f.). ¡Qué vuelva la cultura ciudadana! Bogotá cómo vamos. Recuperado el 4 de marzo de 2021 de https://bogotacomovamos.org/que-vuelva-la-cultura-ciudadana/

Páramo, P. (2017). The city as an environment for urban experiences and the learning of cultural practices. In G. Fleury-Bahi, E. Pol Urrutia, O, Navarro Carrascal (Eds). Handbook of environmental psychology and quality of life research (pp. 275-291). Springer.

Páramo, P. (2013) Comportamiento urbano responsable: las reglas de convivencia en el espacio público. Revista Latinoamericana de Psicología, 45(3), 473-485.

Páramo, P. (2010). Aprendizaje situado: creación y modificación de prácticas culturales en el espacio público urbano. Revista Psicología \& Sociedade, 22(1), 130-138.

Páramo, P., \& Contreras, M. M. (2018). Formación ciudadana en comportamientos urbanos responsables: etnografía rápida orientada a la convivencia en el espacio público urbano. Revista Cuadernos de Vivienda y Urbanismo, 11(22). https://doi. org/10.11144/Javeriana.cvu11-22.fccu

Páramo, P., \& Páramo, J. (2016). Los comportamientos urbanos responsables del peatón. Fundación MAPFRE. https://www.fundacionmapfre.org/fundacion/es es/images/los-comportamientos-urbanos-responsables-del-peaton tcm164127791.pdf

Revista Semana. (2016, junio 8). Bogotá. ¿El regreso de la cultura ciudadana de Mockus? Recuperado el 2 de marzo de 2021 de https://www.semana.com/agenda/articulo/cultura-ciudadana-antanas-mockus-enrique-penalosa/49130/

Ribes, E (1990). Psicología General. México: Trillas

Sandoval, M. (2012). Comportamiento sustentable y educación ambiental: una visión desde las prácticas culturales. Revista Latinoamericana de Psicología, 44(1), 181 196. http://dx.doi.org/10.14349/rlp.v44i1.943

Schultz, P. W., \& Kaiser, F. (2012). Promoting Pro-Environmental Behavior. In S.D. 
Clayton: The Oxford Handbook of Environmental and Conservation Psychology. Oxford University Press. University Press. http://dx.doi.org/10.1093/oxfordhb/9780199733026.013.0029

Schwartz, S. H. (1977). Normative influences on altruism. In L. Berkowitz (Ed.), Advances in experimental social psychology, 10 (pp. 221-279). Academic Press.

Smith, G. S., Houmanfar, R., \& Louis, S. J. (2011). The participatory role of verbal behavior in an elaborated account of metacontingency: From conceptualization to investigation. Behavior and Social Issues, 20, 122- 146. https://doi.org/10.5210/ bsi.v20i0.3662

Todorov, J.C (2013). Conservation and transformation of cultural practices through contingencies and metacontingencies. Behavior and Social Issues, 22, 6473. https://doi.org/10.5210/bsi.v22i0.4812

Todorov, J.C. (2010). Schedules of cultural selection: Comments on "Emergence and Metacontingency". Behavior and Social Issues, 19, 86-89. https://doi.org/10.5210/ bsi.v19i0.3221

Uzzell, D. (1999). Education for environmental action in the community: New roles and relationships. Cambridge Journal of Education, 29(3), 397-413. https://doi. org/10.1080/0305764990290309

Van Houten, R., \& Malenfant, J. E. L. (2004). Effects of a driver enforcement program on yielding to pedestrians. Journal of Applied Behavior Analysis, 37, 351-363. https://doi.org/10.1901/jaba.2004.37-351

Wilson, E.O. (1993). Biophilia: The human bond with other species. Harvard University Press.

Zandecki, E.M. (2012) The effects of informational prompts and performance feedback on recyclign behaviour. Graduate Theses and Dissertations. http://scholarcommons.usf.edu/etd/4265

Zilio, D. (2019). On the Function of Science: an Overview of 30 Years of Publications on Metacontingency. Behavior and Social Issues 28, 46-76 https://doi.org/10.1007/ s42822-019-00006-x 